\title{
Shillourokambos et les debuts du Neolithique à Chypre
}

\author{
Jean Guilaine, François Briois, Jacques Coularou, Jean-Denis Vigne, \\ ISABELLE CARRÈRE
}

\section{RESUMEN}

Los autores aportan verdaderas novedades sobre la primera ocupación del hombre prehistórico en Chipre. Desde el X-IX milenio a $C$. los cazadores recolectores documentados en Akrotiri-Aetokremnos, en el sur de la isla, muestran el aprovechamiento de sus recursos antes del Neolítico. Pero sobre todo, las excavaciones de Shillourokambos han documentado fases del Neolítico precerámico que se remontan a finales del IX milenio y primera mitad del VIII, en fechas calibradas, contemporáneas del precerámico B del Levarite, anteriores al precerámico tipo Khirokitia del VII milenio.

Jusqu'à ces dernières années, la néolithisation de l'île de Chypre revêtait, par rapport à l'aire proche-orientale voisine, des aspects originaux et, semblait-il, de chronologie tardive. Rappelons que Chypre est fréquentée, au cours des $\mathrm{Xe}$ et IXe millénaires avant l'ère, par des chasseurs-collecteurs dont on a reconnu la présence sur le site d'Akrotiri-Aetokremnos, dans la partie la plus méridionale de l'île. Ici une industrie de pierre taillée paraît associée à une économie "mésolithique» de chasse au petit gibier (oiseaux), de pêche et de consommation de mollusques marins. L'idée que ces chasseurs-collecteurs sont également responsables de l'extinction de la faune endémique de l'île s'appuie sur le fait qu'une importante accumulation d'ossements de mammifères (220 hippopotames nains, 3 éléphants nains) a été reconnue sur le même site. Toutefois les liens entre le dépôt d'ossements d'une part et la fréquentation humaine de l'autre ne sont pas indubitablement démontrés et font actuellement l'objet d'un débat qui ne pourra être levé qu'après une analyse plus fine des données ar- 
chéologiques. Un fait demeure: l'homme fréquentait bel et bien l'île avant que les agro-pasteurs n'y installent leurs premiers établissements.

L'implantation des premières communautés agricoles était généralement attribuée à des migrants d'origine continentale dont le plus fort impact semblait jusqu'à peu, à l'obsenvation des dates disponibles, se placer entre 8000 et 7000 BP, c'est-à-dire dans le courant du VIle millénaire en dates calibrées. Vers la fin des années quatre-vingt, on disposait déjà, pour le Néolithique pré-céramique chypriote, d'une quarantaine de dates radiocarbone dont la très grande majorité montraient une nette concentration dans l'intervalle évoqué ci-dessus. Sensiblement «jeune» par rapport à ses voisins proche-orientaux, ce Néolithique "pré-céramique», bien connu grâce à une série d'importantes fouilles sur des sites devenus classiques - Khirokitia, Kalavasos-Tenta, Cap Andreas Kastros, Dhali Agridhi, Trouilli-y affecte aussi des caractères spécifiques qui contribuent à marquer sa "distance culturelle" avec le continent voisin. Ainsi l'architecture circulaire semble s'opposer aux maisons rectangulaires alors bien implantées au Levant. Les industries de la pierre, culturellement peu typées, n'y connaissent pas la production de pièces de qualité (et notamment des armatures de projectile) telles que peuvent les générer, à la même époque, les cultures de type "PPNB" (Néolithique pré-céramique B du Levant et d'Anatolie). L'élevage du mouton, de la chèvre et du porc est pratiqué, à Chypre comme au Levant, grâce à l'introduction de ces espèces sur l'île, mais celui du boeuf, pourtant bien attesté sur le continent à cette époque, est absent et n'apparaîtra qu'à l'Age du Bronze. De même, au Vlle millénaire, Chypre ignore toujours la céramique, déjà présente, sur le littoral, de la Cilicie jusqu'au Liban.

De toutes récentes recherches entreprises sur un site méridional de l'île - Shillourokambos à Parreklisha- sont en passe de nuancer ce tableau, voire de le compléter en apportant de nouvelles lueurs sur des phases antérieures du Néolithique chypriote.

\section{UNE FORTE DENSITÉ DE SITES NÉOLITHIQUES}

Tout commence vers la fin des années quatre-vingt, lorsque Pierre Aupert, responsable de la fouille d'Amathonte, à quelques kilomètres à l'est de Limassol, décide de faire dresser la carte archéologique de l'arrière-pays amathousien. II s'agit au départ d'évaluer le peuplement du "Royaume d'Amathonte" et les relations entre une cité et son territoire rural. Réalisées par Catherine Petit et son équipe, ces prospections ne se limitent pas aux sites antiques et sont menées toutes périodes confondues. Elles révélent un nombre particulièrement élevé de sites néolithiques témoignant dans 


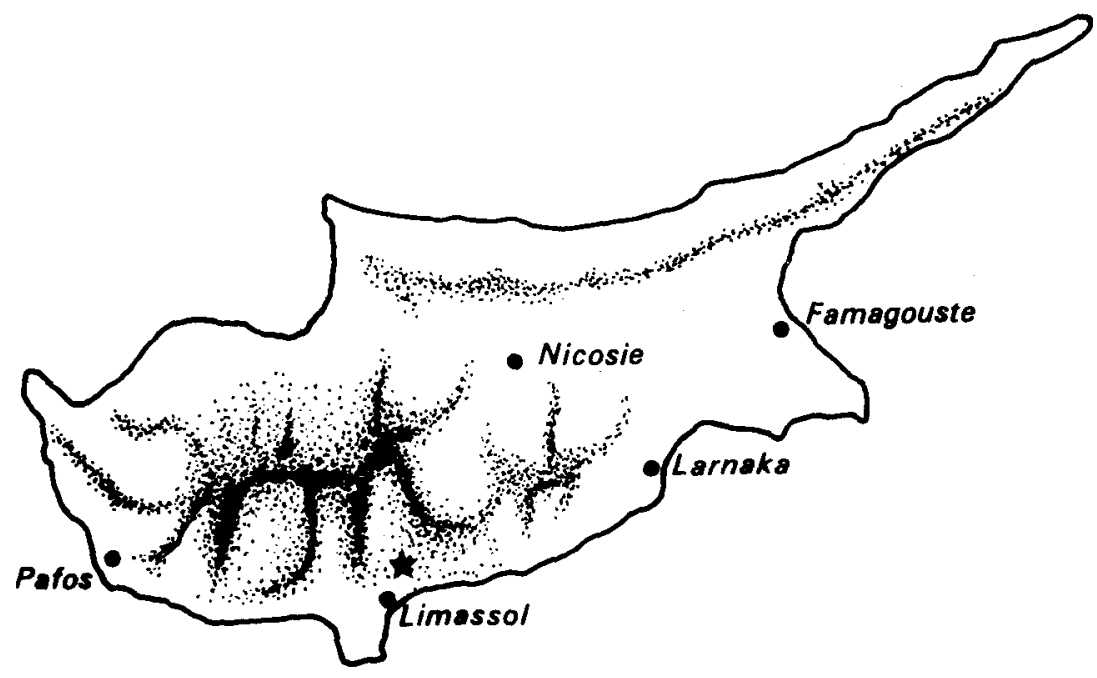

Figure 1. Localisation (étoile) du site de Shillourokambos (Parekklisha) à l'Est de Limassol.

cette région de l'île d'une sédentarisation ancienne et conséquente. En 1991 et 1992 a lieu sur le terrain une révision de tous les gisements reconnus; elle permet d'apprécier les potentialités de chacun d'eux et d'en estimer sommairement la position chrono-culturelle. Un site retient vite notre attention. Shillourokambos, aujourd'hui situé à quelque $5 \mathrm{~km}$ à l'intérieur des terres, sorte de petit plateau encadré par deux vallons. Sa taille d'abord nous surprend: les découvertes de surface s'étalent sur 4 hectares, mais il est vraisemblable que ces dimensions sont surestimées en raison d'épandages dus à la déclivité du terrain vers le sud. Les ramassages ensuite montrent la présence de vestiges se rapportant à la phase pré-céramique comme à la culture néolithique de Sotira (Ve millénaire): il existe donc ici la possibilité de disposer d'une occupation stratifiée sur une certaine durée. Divers sondages témoignent d'une sédimentation par rapport au sol actuel de puissance variable, souvent peu fournie $-0,60$ a $0,80 \mathrm{~m}$ mais pouvant atteindre jusqu'à 1,80 $\mathrm{m}$ (Sondage 2)-. L'aire la plus sédimentée révèle en particulier une superposition de niveaux autorisant l'élaboration d'une chronologie à l'intérieur du «pré-céramique».

Depuis les recherches entreprises entre 1993 et 1996 -qui comportent notamment le décapage d'une aire de plus de $800 \mathrm{~m}^{2}$ - permettent, bien qu'embryonnaires, de présenter aujourd'hui un visage sensiblement renouvelé de la néolithisation de Chypre. 


\section{UNE NÉOLITHISATION PLUS ANCIENNE QUE PRÉVU}

Si le Vlle millénaire concentre, on l'a vu, la majorité des datations du Pré-céramique, quelques dates absolues plus anciennes avaient été proposées il y a quelques années pour les niveaux inférieurs du site de Tenta. Certains avaient alors avancé l'idée d'une apparition plus précoce du Néolithique à Chypre. Malheureusement ces datations étaient parfois grévées par des intervalles de confiance assez considérables ( \pm 400 à 500 ans) ce qui les rendait peu utilisables. Or ce qui n'était qu'hypothèse reçoit, avec le site de Shillourokambos, confirmation. II a existé à Chypre des phases pré-céramiques très sensiblement antérieures au VIle millénaire.

A ce jour la datation la plus précoce du site (Secteur 1, Couche 2) accuse $9310 \pm 80 \mathrm{BP}$ (soit, après correction, les derniers siècles du IXe millénaire). On observera qu'elle est un peu plus ancienne que la date de $9240 \pm 130$ BP qui marque, à Kalavasos, l'étape initiale de l'établissement de Tenta. Quatre autres dates à Shillourokambos (Secteur 1, Couches 1 et $1 / 2$ ) se placent, pour le Pré-céramique ancien chypriote, entre $8824 \pm 100$ $\mathrm{BP}$ et $8655 \pm 65 \mathrm{BP}$, soit dans la première moitié du VIlle millénaire, en datations calibrées.

Plusieurs de ces dates ont de faibles marges statistiques, ce qui conforte leur fiabilité. L'industrie et l'architecture en particulier confirment cette antiquité qui fait actuellement de Shillourokambos le plus vieux site néolithique de Chypre. II a donc existé sur cette île un peuplement néolithique plus ou moins contemporain du Néolithique pré-céramique $B$ «moyen» du Levant et c'est donc avec les cultures de la phase moyenne du PPNB qu'il faudra désormais comparer les données chypriotes.

II s'ensuit que les sites “classiques» du Pré-céramique, tels que Khirokitia et Cap Andreas Kastros, centrés sur le Vlle millénaire, correspondent au plein développement d'une civilisation qui puise ses racines dans les siècles antérieurs. De fait, à cette époque, le Néolithique chypriote a déjà, derrière lui, une longue histoire.

\section{LA QUESTION DES ORIGINES CONTINENTALES}

Au plan chronologique, Shillourokambos pose donc en termes nouveaux la question des relations avec le continent. II s'ensuit que l'un des problèmes à résoudre consistera à essayer de déchiffrer les savoirs techniques "continentaux" qui ont pu passer sur l'île, lors du transfert des populations néolithiques initiales. Or les premières observations en ce do- 

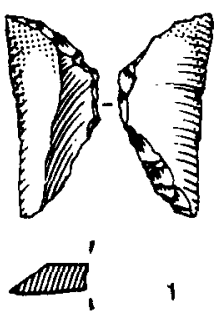
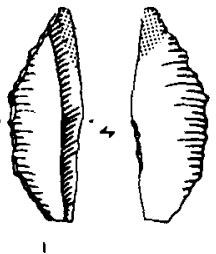

2
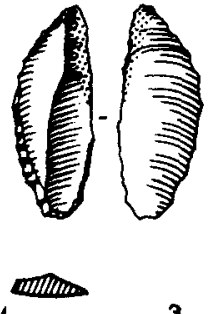

3
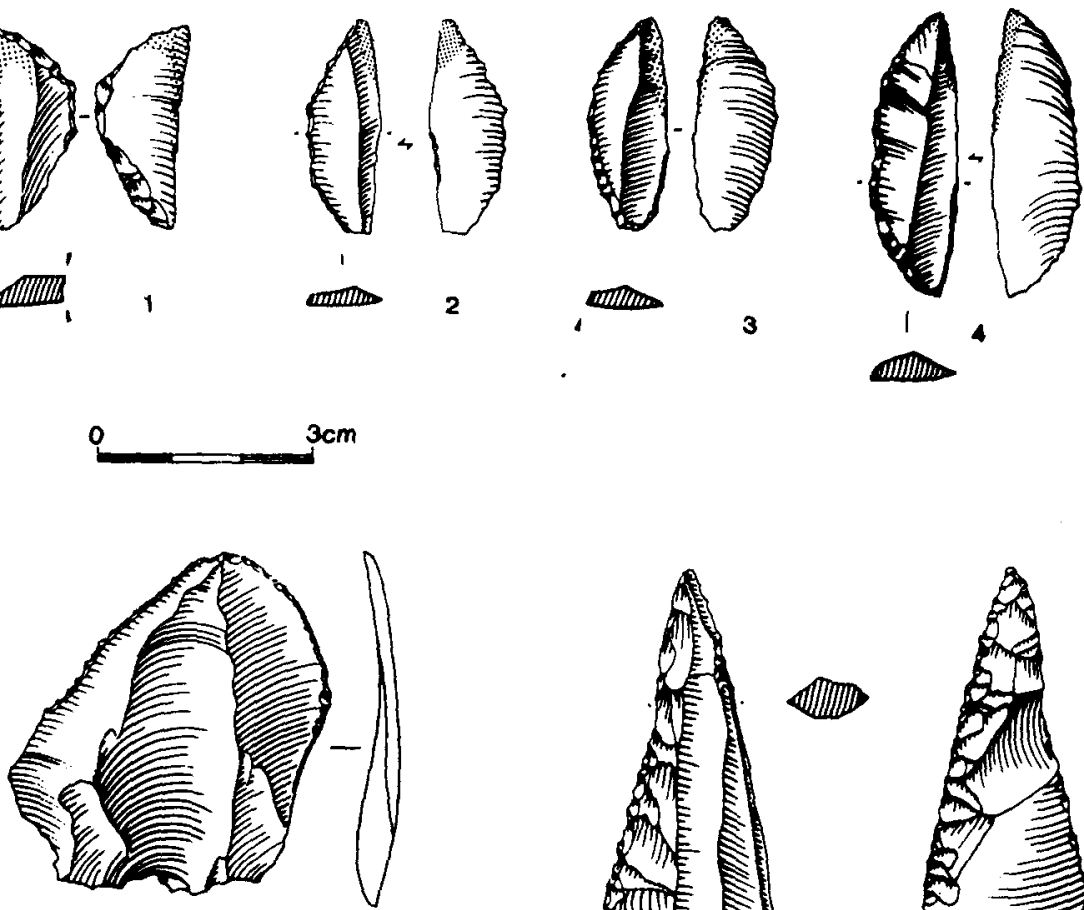

5
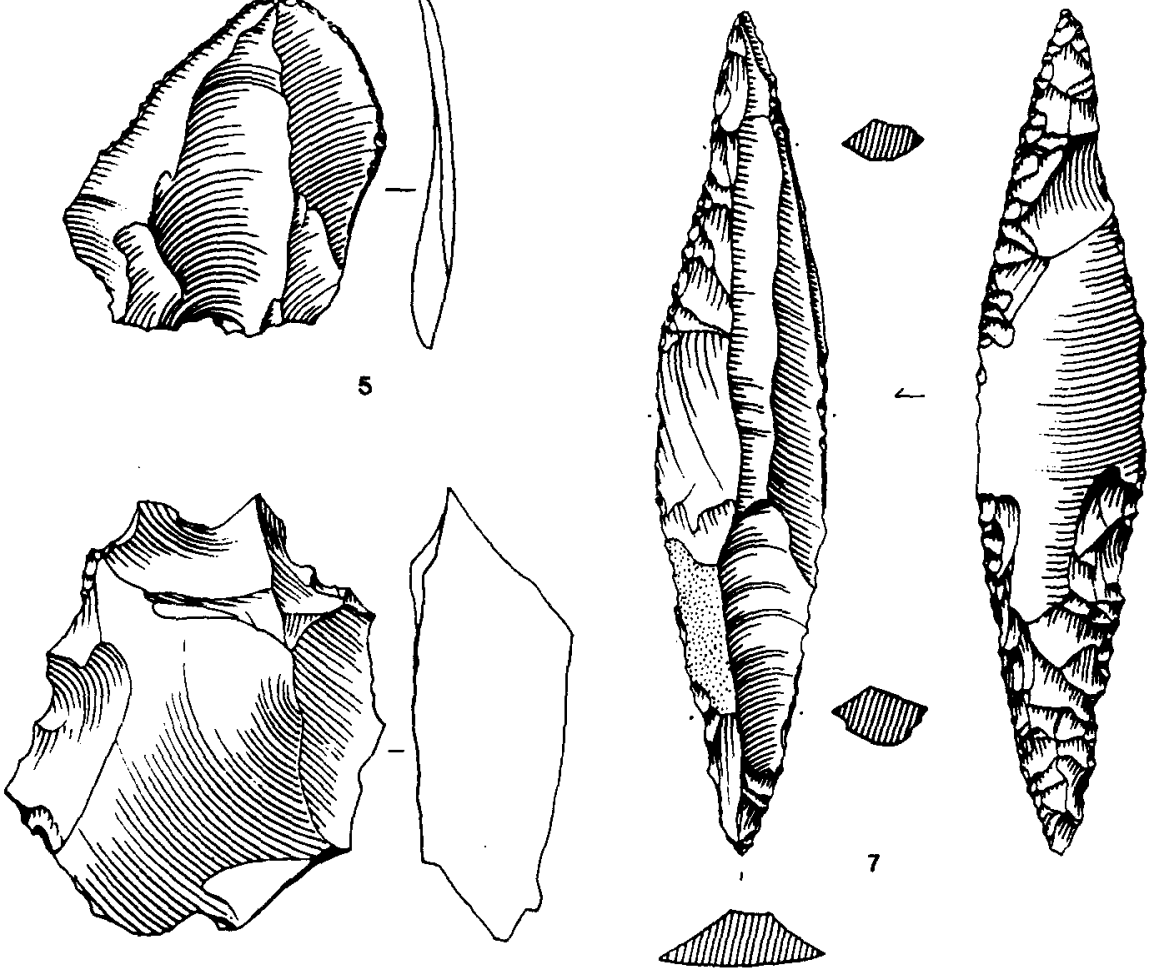

6

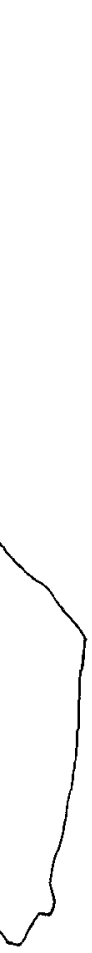

cising

Figure 2. Shillourokambos. Pré-céramique. Phases anciennes. Secteur 1-C1.

1 à 4: géométriques à dos abattu et à lustré oblique (armatures de faucille); 5: grattoir sur éclat mince; 6: pièce à coches multiples; 7: pointe de flèche à retouches bifaciales. (Dessin F. Briois). 
maine montrent quelques correspondances intéressantes. A côté d'une abondante production d'éclats, d'ailleurs permanente tout au long du Précéramique, les séries chypriotes anciennes montrent le recours, dans la production de lames, à une technique propre au «PPNB»: le débitage bipolaire, réalisé à partir de nucléus naviformes, et qui permet d'obtenir des lames de formes prédéterminées à extrémité pointue. Ces dernières seront, entre autres, sélectionnées pour la fabrication d'armatures de flèches à extrémités retouchées. Les quelques pointes de projectile mises au jour semblent également conforter cette filiation avec le continent, dont nous ignorons de façon précise la (ou les) souche géographique.

Dans cette approche un autre point mérite attention: les éléments en obsidienne sont bien représentés dans les dépôts anciens du site, tout en restant statistiquement faibles comparativement à l'industrie de silex. Mais cette présence est, qualitativement, intéressante. Les sites pré-céramiques de l'île n'ont, en général, fourni qu'un nombre limité de pièces en obsidienne (30 à 40 pièces au maximum). Or, à Shillourokambos, après des travaux n'ayant entraîné qu'un traitement de sédiments limité, l'on dispose déjà de plus de 200 pièces, ce qui en fait, d'assez loin, le site pré-céramique le plus fourni de l'île en cette matière. Tous ces éléments - presque toujours des lamelles obtenues par "pression"- sont d'origine anatolienne et les analyses de Bernard Gratuze, du Centre E. Babelon du CNRS, situent en Cappadoce, autour des volcans du Gollu Dag et du Nenezi Dag, leur aire d'origine. Il est surtout intéressant de constater que ce trafic de l'obsidienne est caractéristique des étapes anciennes du Pré-céramique, celles où les échanges maritimes semblent avoir connu leur plus grande intensité avec les processus de néolithisation de l'île. On ignore si ces importations sont le fruit de contacts directs avec les côtes sud-anatoliennes ou si elles ont transité par le Levant, la circulation de l'obsidienne de la Cappadoce vers l'aire syro-palestinienne constituant un circuit de distribution ancien, remontant à l'Epipaléolithique.

II serait tentant mais peu fondé, en l'état, d'affirmer que le site était un "centre de redistribution" en raison du nombre de lamelles fourni. Lorsque l'on aura fouillé d'autres établissements de cette haute époque, il sera possible d'établir des comparaisons et de juger si cette relative fréquence est ici normale ou confère à l'établissement un statut particulier.

Parmi les productions d'outils de silex, on signalera la présence de segments de cercle à dos abattu et à lustré oblique, très probablement des éléments composites de faucilles. 

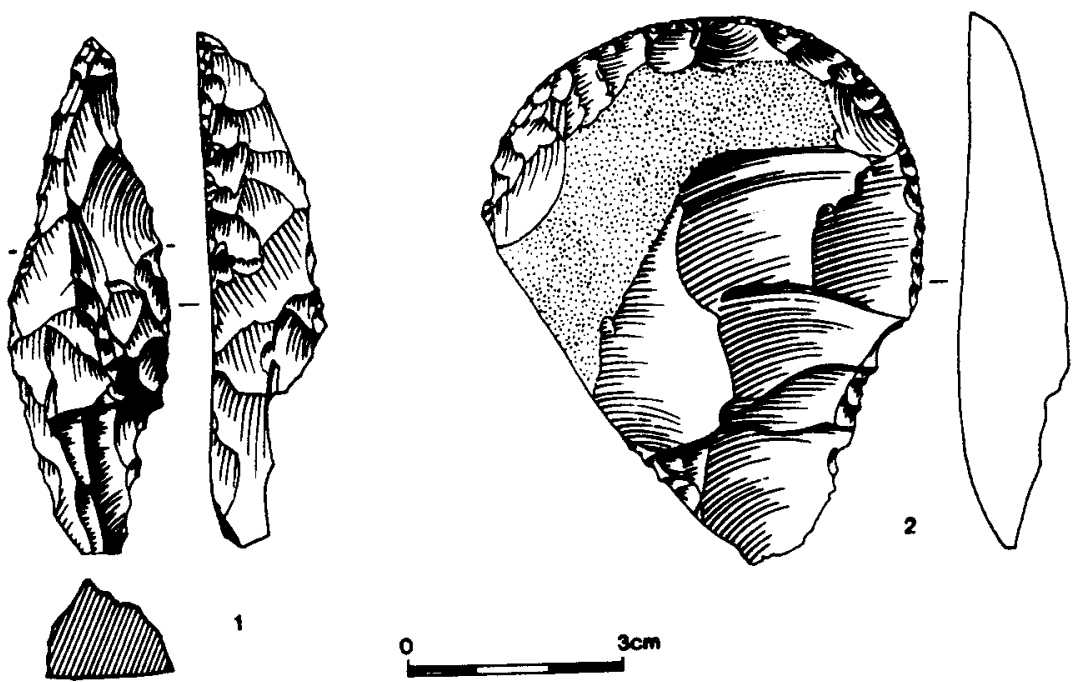

1
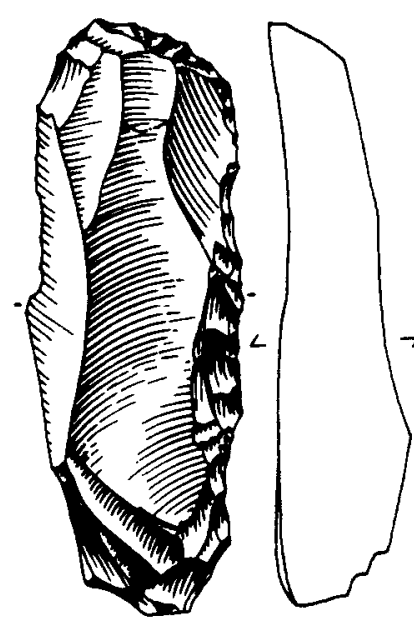

3

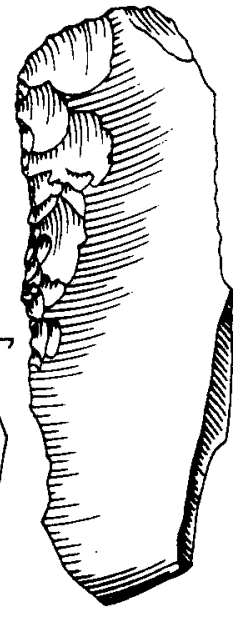

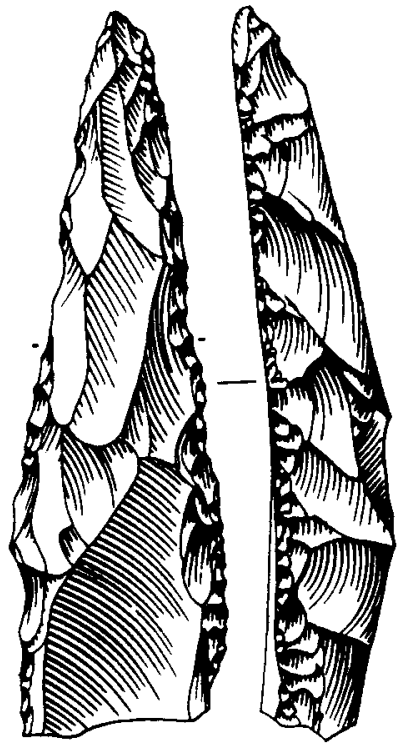

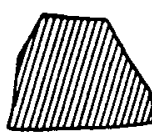

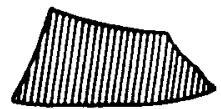

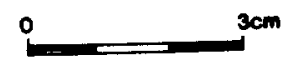

Shillourokambos. Pré-céramique. Phases récentes. Sondage 2 - C3.

1: bec triédrique sur lame; 2: grattoir régulier sur éclat mince; 3: grattoir sur bout de lame retouchée; 4: lame appointée par retouches abruptes. (Dessin F. Briois). 


\section{PEUT-ON «PÉRIODISER» LE PRÉ-CÉRAMIQUE CHYPRIOTE?}

Shillourokambos permet d'introduire quelques nuances dans l'évolution interne du Néolithique pré-céramique. Un sondage stratifié et diverses variations géographiques, à l'intérieur du site, autorisent pour l'instant un découpage en trois ou quatre étapes fondées sur l'analyse des industries.

L'étape ancienne, que l'on subdivisera en une phase A (Secteur 1 , Couche 2) et en une phase $B$ (Secteur 1 , couches 1 et $1 / 2$ ), fait essentiellement appel' à une variété de chert translucide d'excellente qualité. Ce matériau, issu de gîtes éloignés du gisement, est introduit sous la forme de grands éclats utilisés pour la mise en forme et l'exploitation de nucléus à lames ou à éclats. Le débitage laminaire bipolaire à partir de nucléus naviformes prend alors un relief tout particulier. Les outils se composent de pièces à coches, de racloirs, de grattoirs, etc. Ces niveaux ont livré les quelques pointes de projectile connues, des segments de cercle pour armer des faucilles, la plupart des pièces en obsidienne.

La période moyenne d'occupation (Secteur 1, Couche 0) voit apparaître les formes d'une mutation dans les industries. Le chert opaque, abondant et accessible à faible distance du site, devient prépondérant. Les lames produites sont alors plus grandes, plus robustes aussi mais conservent toujours le concept de débitage bipolaire. La production d'outils sur éclats se poursuit (grattoirs, perçoirs, becs, mèches de forets) et les géométriques à lustré oblique laissent la place à des couteaux à moissonner sur lame dont le poli devient désormais longitudinal.

L'étape la plus récente datée dans le secteur 4 " maison 1 " de $8125 \pm$ 70 BP connaît l'emploi exclusif du chert opaque utilisé pour la production d'outils sur éclats et sur lames robustes obtenues en percussion. Une notable simplification des concepts technologiques précédents reflète une mutation dans la taille du silex peu de temps avant les étapes classiques reconnues sur l'île, notamment à Khirokitia et à Cap Andreas Kastros.

Tout au long de la séquence l'outillage lourd évolue peu: percuteurs sphériques ou discoïdes, percuteurs sur galets allongés, meules, molettes, haches polies. Les contenants sont, presque toujours, en calcaire (bols, godets, bassins biconiques). Il existe des mortiers peu profonds obtenus par piquetage de gros blocs ou de galets. On n'observe pas d'évolution sensible dans la morphologie des récipients. 


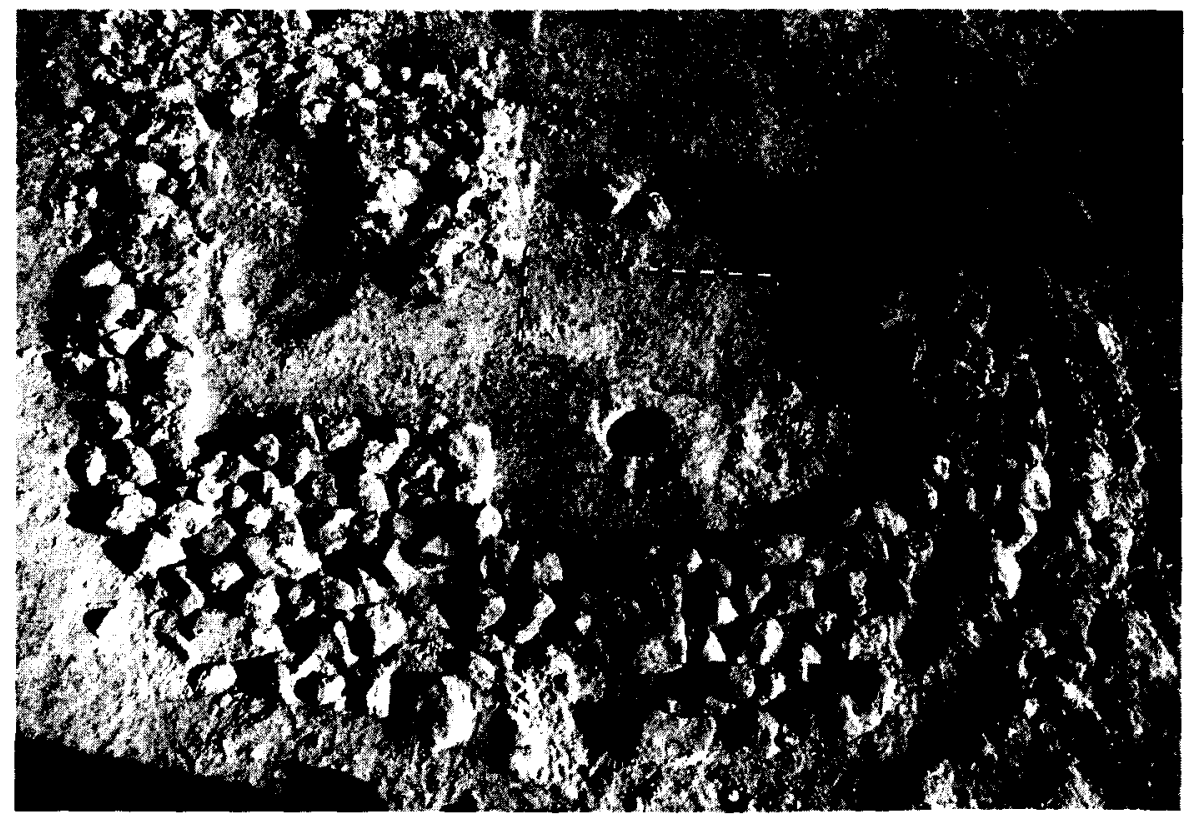

Figure 4. Shillourokambos. Pré-céramique. Phases récentes. Secteur 4.

Restes d'un bâtiment rond, bâti par juxtaposition de blocs massifs et comportant, à l'intérieur, l'assise d'un appendice construit ainsi qu'un trou de poteau; on peut voir, à l'extérieur du bâtiment précédent, un tronçon de mur curviligne construit dans un appareil de moindre volume et attribuable à une architecture distincte. Vue prise du Nord-Ouest.

(Cliché J. Coularou).

\section{LES CARACTÈRES DE L'HABITAT ANCIEN}

Quand s'amorce à Shillourokambos l'architecture circulaire en pierre? Elle est présente, on l'a vu, lors de la phase terminale du site mais nul doute qu'elle ne débute plus précocement. En tout cas, les étapes les plus anciennes montrent, quant à elles, une architecture totalement différente, matérialisée par l'usage de poteaux aux assises creusées dans le substrat ou par des tranchées insérant elles-mêmes des emplacements de pièces de bois. On connaît ainsi des alignements de poteaux formant, dans un cas, une double file. Cette phase ancienne à architecture de bois avait aussi été observée à Kalavasos-Tenta (phase 5 de Todd) mais, fouillée sur une superficie trop restreinte, n'avait pu donner lieu à une interprétation développée.

Mais ce sont les constructions sur tranchées qui font l'originalité de Shillourokambos lors de cette étape ancienne. En 1994, nous avions ainsi 
dégagé un curieux "bâtiment" triangulaire (ou sub-trapézoïdal), à deux portes étroites, de un mètre de large, chacune encadrée par deux trous de poteaux. Cet aménagement s'étirait sur $19 \mathrm{~m}$ de longueur pour $8 \mathrm{~m}$ de largeur maximum et délimitait un espace de $76 \mathrm{~m}^{2}$. Cette architecture étonnante n'était en fait nullement isolée mais s'intégrait à un enclos au tracé curviligne: le grand côté du bâtiment constituait un tronçon de cette plus vaste installation, en apparence sub-circulaire et dégagée aujourd'hui sur plus de $30 \mathrm{~m}$ de développement.

D'autres agencements de cette variété ont pu être observés un peu plus à l'est. L'un, dégagé aujourd'hui sur plus de $25 \mathrm{~m}$, décrit une sorte de tracé sinueux; il semble avoir été doublé par plusieurs petites tranchées sub-parallèles dont subsistent quelques tronçons. Un second dispositif, long d'une vingtaine de mètres, décrit un arc ovale et se termine peu après une étroite interruption.

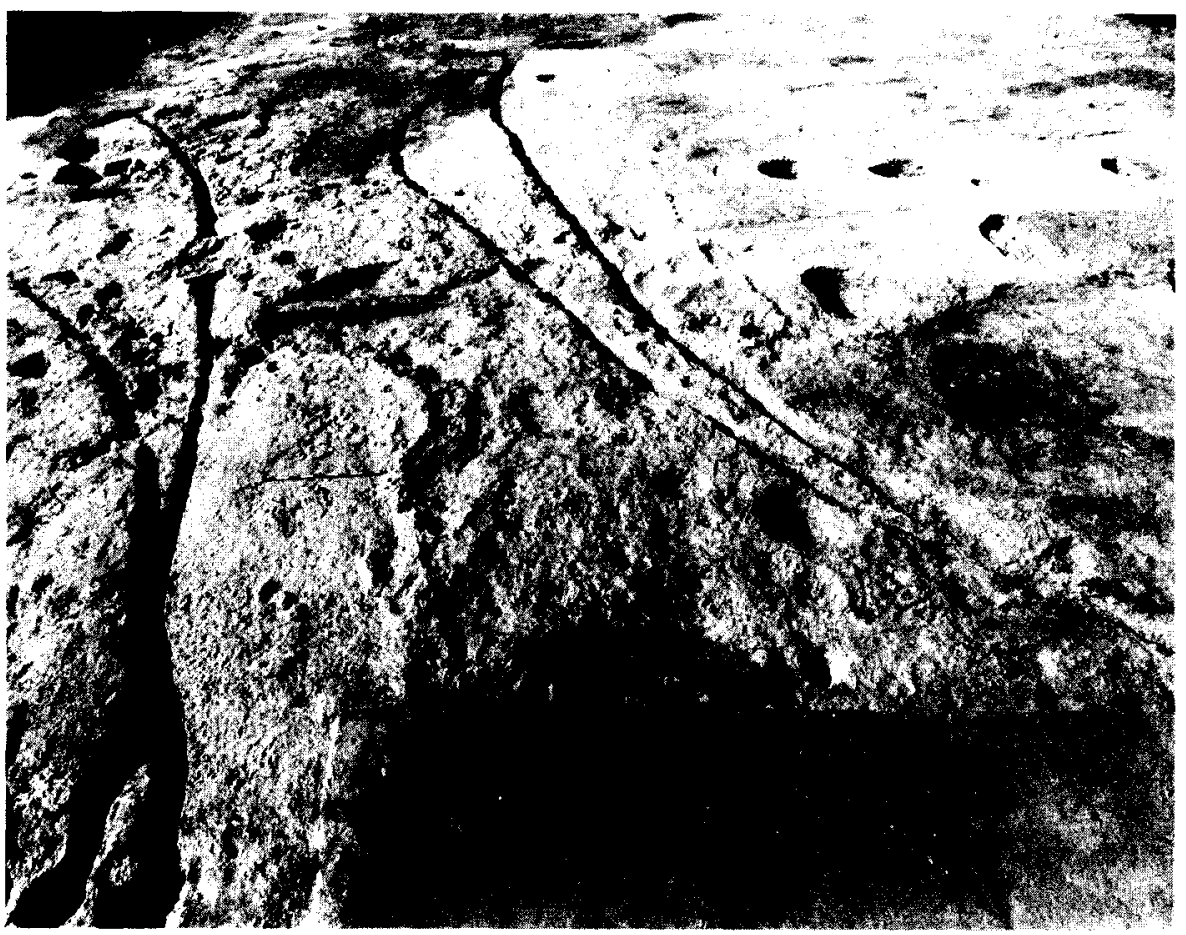

Figure 5. Shillourokambos. Pré-céramique. Phases anciennes. Vue générale sur le Secteur 1 "Nord", prise de l'Ouest. On distingue les tranchées de palissade des "complexes" oriental et occidental ainsi que les nombreuses structures creusées dans le substrat. (Cliché J. Coularou). 
A quoi pouvaient servir ces dispositifs palissadés? Délimitaient-ils des espaces destinés à parquer le bêtes ou leur fonction était-elle plus comple$x e$ ? Peut-être abritaient-ils plusieurs aires domestiques complémentaires: lieux d'habitat, zones d'activités diverses, étables, bergeries ou soues, un peu tout à la fois? II est possible, comme le suggèrent certains trous de poteaux ou de piquets dont quelques uns alignés, qu'il ait existé une sorte de découpage interne de ces espaces. Auraient été ainsi juxtaposées des aires consacrées à l'habitat, des greniers, des secteurs dévolus au parcage des bêtes. A l'intérieur de ces zones ceinturées, on trouve aussi des structures de combustion (rares), mais, surtout, des sortes de cuvettes cylindriques, aux profondeurs diverses, peut-être des agencements pour la conservation de denrées ou, pour les plus profondes, des sortes de citernes.

Si ces "palissades murs " épousent le plus souvent un tracé curviligne, elles montrent aussi l'existence de tronçons rectilignes, voire l'usage de l'angle droit parfaitement maîtrisé (ainsi à la jonction des tranchées 1 et 2). Plusieurs savoirs ou traditions semblent ainsi mémorisés. II n'est pas interdit de penser que ces plans souvent à tendance circulaire aient constitué les prototypes des architectures rondes dont les néolithiques chypriotes feront, peu après, un si large usage.

\section{DES BOVINS QUI DISPARAISSENT ET UN CLIN D'OEIL AUX PREMIËRES DOMESTICATIONS}

Jusqu'à présent, les recherches conduites sur les sites pré-céramiques de Chypre avaient essentiellement mis au jour, parmi les espèces consommées, des caprins (chèvres et moutons), du porc et du daim. Toutes ces espèces ont été introduites par l'homme au début du Néolithique sans doute sous la forme domestique pour les premières. Quelques indices, qu'il faudra bien sûr confirmer, semblent indiquer que le daim, en revanche, a été introduit sans avoir été domestiqué à proprement parler, et pour être chassé. C'est là une illustration de plus, mais sans doute la plus ancienne connue, de l'importance symbolique, voire sociale, que les néolithiques accordaient à la chasse aux cervidés, au point de l'implanter, dès les phases initiales, sur les territoires nouvellement colonisés, notamment les îles où les cerfs manquaient.

Les bovins n'ont jamais été signalés avant le Bronze ancien : aucun site antérieur au llle millénaire n'en a fourni de traces... jusqu'aux travaux entrepris à Shillourokambos. Les phases anciennes de l'établissement ont en effet livré des ossements de bovins en nombre réduit certes mais significatif (en 1995, 9\% du nombre des restes fauniques en C1, Secteur 1). C'est l'indice que le boeuf domestique figurait bel et bien à l'origine parmi 
les espèces introduites et élevées sur l'île par les agro-pasteurs. Dès lors se pose un problème tout aussi passionnant: pourquoi l'espèce a-t-elle disparu au bout de quelques siècles comme semble l'indiquer la très grande rareté d'ossements de boeuf dès la transition aux phases d'occupation évoluées du site de Shillourokambos? Faut-il envisager des raisons anthropiques, écologiques, épidémiques, idéologiques? Le débat est ouvert.

Autre intérêt également de Shillourokambos : en démontrant la présence du boeuf et du porc domestique dès $8200 / 8000$ avant J.-C., ce site fournit parmi les plus anciennes traces de leur état domestique au ProcheOrient. Curieusement c'est donc le domaine insulaire, où l'évidence du statut domestique du boeuf est manifeste en raison de l'absence locale d'ancêtre sauvage, qui documente sur l'ancienneté du processus. Sur le continent, en effet, la proximité morphologique de l'aurochs et des premiers boeufs rend la reconnaissance chronologique de l'émergence de la domestication particulièrement ardue.

\section{DES BATTERIES DE SILOS}

L'histoire de Shillourokambos ne prend pas fin avec les derniers siècles du VIlle millénaire. Le site est réoccupé au Ve millénaire par les populations de la culture de Sotira, la grande civilisation chypriote du plein Néolithique, avec poterie cette fois. De l'habitat potentiel, rien n'est parvenu jusqu'à nous. Par contre de nombreux silos groupés sur la partie au nord du site, plus clairsemés vers le sud, ont été creusés dans le substrat rocheux. Dans les aires de plus forte densité, ces silos ont malheureusement endommagé voire totalement détruit les aménagements du Pré-céramique. Ainsi les «palissades" des grands enclos circulaires sont-elles, vers le nord, complètement démantelées par ces excavations de fort volume pouvant atteindre, pour les plus grandes, 2,50 $\mathrm{m}$ de diamètre. Leur comblement secondaire, qui a transformé ces silos en dépotoirs, associe de nombreux blocs à un sédiment jaune grisâtre, fin, voire, le plus souvent, franchement gris ou noirâtre et à du matériel archéologique: galets de rivière bruts ou aménagés, bouches de fermeture de vases en pierre découpée, industrie lithique taillée dont des couteaux à moissonner, fragments de vases de pierre, restes de bouteilles ou de jattes en céramique à engobe externe rouge vif ou brun brillant, restes de récipients de terre cuite à surfaces peu élaborées.

Cette présentation du site, en cours d'étude, de Shillourokambos montre que ce sont essentiellement les phases précoces du Néolithique pré-cé- 
ramique qui apportent les données les plus neuves sur l'implantation à Chypre des premières communautés agro-pastorales. C'est au cours de ces lointaines étapes, contemporaines ou fort proches dans le temps de la colonisation de l'île par les producteurs, que se mettent en place les traits spécifiques du premier Néolithique chypriote. Certains caractères répercutent manifestement des échos continentaux: le débitage de lames par technique bipolaire à partir de nucléus naviformes, les armatures de projectile à pointe et base retouchées, la circulation de l'obsidienne anatolienne en sont des témoignages. D'autres ont une saveur plus originale: l'architecture sur poteaux, les espaces enclos, le rôle des cervidés -ici, le daim- introduits pour conserver à la chasser un certain poids socio-symbolique, préfigurent déjà une identité insulaire qui culminera, quelques siècles plus tard, avec la culture de Khirokitia. 
